Supporting Information

${ }^{1} \mathrm{H}$ NMR data $\left(\mathrm{CDCl}_{3} / \delta\right)$ and yields of the polymers. XRD pattern of polymer-4a(6/4-OEH). Effects of $\mathrm{NaOH}$ on light emission of polymer-4a(6/4-OEH) and polymer-4b(6/4-OEH). ${ }^{13} \mathrm{C}\left\{{ }^{1} \mathrm{H}\right\}$ NMR spectrum of the model compound $\mathbf{1}$. Photoluminescence spectra of a mixture of polymer-4a(6/4-OEH) and $\left[\mathrm{Al}\left(\mathrm{q}^{\prime}\right)_{2}(\mathrm{q})\right] 2$ (in a $1: 1$ molar ratio) in $\mathrm{CHCl}_{3}$ and in cast film.

\title{
Main-chain Type 8-Quinolinol Polymers: Synthesis, Optical Properties, and Complex Formation with Metals
}

Takayuki Iijima, Shin-ichi Kuroda, and Takakazu Yamamoto*

Chemical Resources Laboratory, Tokyo Institute of Technology, 4259 Nagatsuta, Midori-ku, Yokohama 226-8503, Japan and Faculty of Engineering, Gunma University, 1-5-1 Tenjin-cho, Kiryu-shi, Gunma, 376-8515, Japan 
${ }^{1} \mathrm{H}$ NMR $\left(\mathrm{CDCl}_{3} / \delta\right)$ data and yield of the polymers.

polymer-1a(6/4-OC12), Yield: 79\%. 8.82 (0.8H, 2-H-Q, 4-H-Q), 7.95 (0.4H, 6-H-Q), 7.47 (0.4H, 3-H-Q), 7.01 (3.2H, Ar-H), 4.09 (6.4H, $\left.-\mathrm{OCH}_{2}\right), 1.86-1.25\left(32 \mathrm{H}, \mathrm{CH}_{2}\right), 1.13$ (3.6H, tert- $\mathrm{Bu}), 0.87\left(9.6 \mathrm{H},-\mathrm{CH}_{3}\right), 0.39\left(2.4 \mathrm{H},-\mathrm{Si}\left(\mathrm{CH}_{3}\right)_{2}\right)$.

polymer-1b(6/4-OC12), Yield: 91\%. 8.92 (0.4H, 2-H-Q), 8.19 (0.4H, 4-H-Q), 7.61 (0.4H, 5-H-Q), 7.27 (0.4H, 7-H-Q), 7.05 (3.2H, Ar-H), 4.03 (6.4H, - $\left.\mathrm{OCH}_{2}\right), 1.86-1.25$ $\left(32 \mathrm{H}, \mathrm{CH}_{2}\right), 1.07(3.6 \mathrm{H}$, tert-Bu $), 0.85\left(9.6 \mathrm{H},-\mathrm{CH}_{3}\right), 0.38\left(2.4 \mathrm{H},-\mathrm{Si}\left(\mathrm{CH}_{3}\right)_{2}\right)$

polymer-1c(6/4-OC12), Yield: 63\%. 8.89 (0.4H, 2-H-Q), 8.20 (0.4H, 4-H-Q), 7.67 (0.4H, 5-H-Q), $7.21(0.4 \mathrm{H}, 7-\mathrm{H}-\mathrm{Q}), 7.02(3.2 \mathrm{H}, \mathrm{Ar}-\mathrm{H}), 4.01\left(6.4 \mathrm{H},-\mathrm{OCH}_{2}\right), 1.86-1.25$ $\left(32 \mathrm{H}, \mathrm{CH}_{2}\right), 1.12\left(3.6 \mathrm{H}\right.$, tert-Bu), $0.86\left(9.6 \mathrm{H},-\mathrm{CH}_{3}\right), 0.37\left(2.4 \mathrm{H},-\mathrm{Si}\left(\mathrm{CH}_{3}\right)_{2}\right)$

polymer-1a(8/2-OC12), Yield: 89\%. 8.82 (0.4H, 2-H-Q, 4-H-Q), 7.94 (0.2H, 6-H-Q), 7.52 (0.2H, 3-H-Q), 7.01 (3.6H, Ar-H), $4.03\left(7.2 \mathrm{H},-\mathrm{OCH}_{2}\right), 1.86-1.25\left(36 \mathrm{H}, \mathrm{CH}_{2}\right), 1.13$ $(1.8 \mathrm{H}$, tert $-\mathrm{Bu}), 0.87\left(10.8 \mathrm{H},-\mathrm{CH}_{3}\right), 0.39\left(1.2 \mathrm{H},-\mathrm{Si}\left(\mathrm{CH}_{3}\right)_{2}\right)$.

polymer-1b(8/2-OC12), Yield: 91\%. 8.91 (0.2H, 2-H-Q), $8.18(0.2 \mathrm{H}, 4-\mathrm{H}-\mathrm{Q}), 7.58$ (0.2H, 5-H-Q), 7.33 (0.2H, 7-H-Q), 7.01 (3.6H, Ar-H), $4.03\left(7.2 \mathrm{H},-\mathrm{OCH}_{2}\right), 1.85-1.25$ $\left(36 \mathrm{H}, \mathrm{CH}_{2}\right), 1.09(1.8 \mathrm{H}$, tert-Bu$), 0.87\left(10.8 \mathrm{H},-\mathrm{CH}_{3}\right), 0.30\left(1.2 \mathrm{H},-\mathrm{Si}\left(\mathrm{CH}_{3}\right)_{2}\right)$

polymer-1a(6/4-OCEH), Yield: 85\%. 8.84 (0.4H, 2-H-Q), 8.77 (0.4H, 4-H-Q), 7.95 (0.4H, 6-H-Q), 7.53 (0.4H, 3-H-Q), $7.00(3.2 \mathrm{H}, \mathrm{Ar}-\mathrm{H}), 3.91\left(6.4 \mathrm{H},-\mathrm{OCH}_{2}\right), 1.82(3.2 \mathrm{H}$ $-\mathrm{CH}), 1.56-1.25\left(32 \mathrm{H}, \mathrm{CH}_{2}\right), 1.13(3.6 \mathrm{H}$, tert-Bu$), 0.98,0.89\left(19.2 \mathrm{H},-\mathrm{CH}_{3}\right), 0.39(2.4 \mathrm{H}$, $\left.-\mathrm{Si}\left(\mathrm{CH}_{3}\right)_{2}\right)$.

polymer-1b(6/4-OEH), Yield: 77\%. 8.92 (0.4H, 2-H-Q), 8.17 (0.4H, 4-H-Q), 7.58 (0.4H, 5-H-Q), 7.46 (0.4H, 7-H-Q), $7.00(3.2 \mathrm{H}, \mathrm{Ar}-\mathrm{H}), 3.91\left(6.4 \mathrm{H},-\mathrm{OCH}_{2}\right), 1.82(3.2 \mathrm{H}$ $-\mathrm{CH}), 1.56-1.25\left(32 \mathrm{H}, \mathrm{CH}_{2}\right), 1.09(3.6 \mathrm{H}$, tert-Bu$), 0.92,0.89\left(19.2 \mathrm{H},-\mathrm{CH}_{3}\right), 0.30(2.4 \mathrm{H}$, $\left.-\mathrm{Si}\left(\mathrm{CH}_{3}\right)_{2}\right)$.

polymer-1a(8/2-OEH), Yield: 90\%. $8.84(0.2 \mathrm{H}, 2-\mathrm{H}-\mathrm{Q}), 8.74(0.2 \mathrm{H}, 4-\mathrm{H}-\mathrm{Q}), 7.95$ (0.2H, 6-H-Q), 7.49 (0.2H, 3-H-Q), $6.99(3.6 \mathrm{H}, \mathrm{Ar}-\mathrm{H}), 3.98\left(7.2 \mathrm{H},-\mathrm{OCH}_{2}\right), 1.82(3.6 \mathrm{H}$ $-\mathrm{CH}), 1.56-1.25\left(36 \mathrm{H}, \mathrm{CH}_{2}\right), 1.13(1.8 \mathrm{H}$, tert-Bu$), 0.98,0.89\left(21.6 \mathrm{H},-\mathrm{CH}_{3}\right), 0.39(1.2 \mathrm{H}$, $\left.-\mathrm{Si}\left(\mathrm{CH}_{3}\right)_{2}\right)$.

polymer-1b(8/2-OEH), Yield: 97\%. 8.92 (0.2H, 2-H-Q), $8.16(0.2 \mathrm{H}, 4-\mathrm{H}-\mathrm{Q}), 7.57$ (0.2H, 5-H-Q), 7.52 (0.2H, 7-H-Q), $7.00(3.6 \mathrm{H}, \mathrm{Ar}-\mathrm{H}), 3.91\left(7.2 \mathrm{H},-\mathrm{OCH}_{2}\right), 1.82(3.6 \mathrm{H}$ $-\mathrm{CH}), 1.56-1.25\left(36 \mathrm{H}, \mathrm{CH}_{2}\right), 1.09(1.8 \mathrm{H}$, tert-Bu $), 0.97,0.88\left(21.6 \mathrm{H},-\mathrm{CH}_{3}\right), 0.30(1.2 \mathrm{H}$, $\left.-\mathrm{Si}\left(\mathrm{CH}_{3}\right)_{2}\right)$.

polymer-2a(6/4), Yield: $82 \% .8 .85(0.4 \mathrm{H}, 2-\mathrm{H}-\mathrm{Q}), 8.68(0.4 \mathrm{H}, 4-\mathrm{H}-\mathrm{Q}), 8.00(0.4 \mathrm{H}$, 6-H-Q), 7.70-7.51 (9.6H, Fl-H), $7.46(0.4 \mathrm{H}, 3-\mathrm{H}-\mathrm{Q}), 2.00\left(6.4 \mathrm{H},-\mathrm{CH}_{2}\right), 1.25-1.07(32 \mathrm{H}$, $\left.\mathrm{CH}_{2}\right), 1.13\left(3.6 \mathrm{H}\right.$, tert-Bu), $0.82\left(9.6 \mathrm{H},-\mathrm{CH}_{3}\right), 0.62\left(6.4 \mathrm{H},-\mathrm{CH}_{2}\right), 0.39(2.4 \mathrm{H}$, 
$\left.-\mathrm{Si}\left(\mathrm{CH}_{3}\right)_{2}\right)$.

polymer-2b(6/4), Yield: 83\%. $8.97(0.4 \mathrm{H}, 2-\mathrm{H}-\mathrm{Q}), 8.25$ (0.4H, 4-H-Q), 7.70-7.51 (9.6H, Fl-H), 7.67 (0.4H, 5-H-Q), 7.33 (0.4H, 7-H-Q), $2.02\left(6.4 \mathrm{H},-\mathrm{CH}_{2}\right), 1.25-1.07(32 \mathrm{H}$, $\left.\mathrm{CH}_{2}\right), 1.18\left(3.6 \mathrm{H}\right.$, tert-Bu), $0.82\left(9.6 \mathrm{H},-\mathrm{CH}_{3}\right), 0.64\left(6.4 \mathrm{H},-\mathrm{CH}_{2}\right), 0.32(2.4 \mathrm{H}$, $\left.-\mathrm{Si}\left(\mathrm{CH}_{3}\right)_{2}\right)$.

polymer-2a(8/2), Yield: $78 \% .8 .85(0.2 \mathrm{H}, 2-\mathrm{H}-\mathrm{Q}), 8.68(0.2 \mathrm{H}, 4-\mathrm{H}-\mathrm{Q}), 8.00(0.2 \mathrm{H}$, 6-H-Q), (11H, Fl-H, 3-H-Q), $2.01\left(7.2 \mathrm{H},-\mathrm{CH}_{2}\right), 1.25-1.07\left(36 \mathrm{H}, \mathrm{CH}_{2}\right), 1.13(1.8 \mathrm{H}$, tert- $\mathrm{Bu}), 0.82\left(10.8 \mathrm{H},-\mathrm{CH}_{3}\right), 0.62\left(7.2 \mathrm{H},-\mathrm{CH}_{2}\right), 0.40\left(1.2 \mathrm{H},-\mathrm{Si}\left(\mathrm{CH}_{3}\right)_{2}\right)$.

polymer-2b(8/2), Yield: 74\%. 8.97 (0.2H, 2-H-Q), $8.25(0.2 \mathrm{H}, 4-\mathrm{H}-\mathrm{Q}), 7.70-7.51(9.6 \mathrm{H}$, Fl-H), 7.67 (0.2H, 5-H-Q), $7.33(0.2 \mathrm{H}, 7-\mathrm{H}-\mathrm{Q}), 2.02\left(7.2 \mathrm{H},-\mathrm{CH}_{2}\right), 1.25-1.07(36 \mathrm{H}$, $\left.\mathrm{CH}_{2}\right), 1.18(1.8 \mathrm{H}$, tert-Bu$), 0.82\left(10.8 \mathrm{H},-\mathrm{CH}_{3}\right), 0.64\left(7.2 \mathrm{H},-\mathrm{CH}_{2}\right), 0.32(1.2 \mathrm{H}$, $\left.-\mathrm{Si}\left(\mathrm{CH}_{3}\right)_{2}\right)$.

polymer-3, Yield: 97\%. 9.02 (0.4H, 2-H-Q), 8.82 (0.4H, 4-H-Q), 7.88 (0.4H, 6-H-Q), $7.53(0.4 \mathrm{H}, 3-\mathrm{H}-\mathrm{Q}), 7.01(3.2 \mathrm{H}, \mathrm{Ar}-\mathrm{H}), 4.50\left(1.2 \mathrm{H},-\mathrm{OCH}_{3}\right), 4.03\left(6.4 \mathrm{H},-\mathrm{OCH}_{2}\right)$, 1.86-1.25 (32H, $\left.\mathrm{CH}_{2}\right), 0.87\left(9.6 \mathrm{H},-\mathrm{CH}_{3}\right)$.

polymer-4a(8/2-OC12), Yield: 100\%. 8.84 (0.4H, 2-H-Q, 4-H-Q), 7.91 (0.2H, 6-H-Q), $7.52(0.2 \mathrm{H}, 3-\mathrm{H}-\mathrm{Q}), 7.01(3.6 \mathrm{H}, \mathrm{Ar}-\mathrm{H}), 4.03\left(7.2 \mathrm{H},-\mathrm{OCH}_{2}\right), 1.86-1.25\left(36 \mathrm{H}, \mathrm{CH}_{2}\right), 0.87$ $\left(10.8 \mathrm{H},-\mathrm{CH}_{3}\right)$.

polymer-4b(8/2-OC12), Yield: 96\%. 8.85 (0.2H, 2-H-Q), 8.23 (0.2H, 4-H-Q), 7.67 (0.2H, 5-H-Q), 7.27 (0.2H, 7-H-Q), 7.01 (3.6H, Ar-H), $4.03\left(7.2 \mathrm{H},-\mathrm{OCH}_{2}\right), 1.86-1.25$ $\left(36 \mathrm{H}, \mathrm{CH}_{2}\right), 0.87\left(10.8 \mathrm{H},-\mathrm{CH}_{3}\right)$.

polymer-4a(6/4-OEH), Yield: 81\%. 8.84 (0.4H, 2-H-Q), 8.78 (0.4H, 4-H-Q), 7.92 (0.4H, 6-H-Q), 7.53 (0.4H, 3-H-Q), $7.00(3.2 \mathrm{H}, \mathrm{Ar}-\mathrm{H}), 3.91\left(6.4 \mathrm{H},-\mathrm{OCH}_{2}\right), 1.82(3.2 \mathrm{H}$ $-\mathrm{CH}), 1.56-1.25\left(38.4 \mathrm{H}, \mathrm{CH}_{2}\right), 0.96,0.88\left(19.2 \mathrm{H},-\mathrm{CH}_{3}\right)$.

polymer-4b(6/4-OEH), Yield: $86 \% .8 .84(0.4 \mathrm{H}, 2-\mathrm{H}-\mathrm{Q}), 8.22(0.4 \mathrm{H}, 4-\mathrm{H}-\mathrm{Q}), 8.05$ (0.4H, OH), 7.52 (0.4H, 5-H-Q), 7.27 (0.4H, 7-H-Q), 7.00 (3.2H, Ar-H), $3.91(6.4 \mathrm{H}$, $\left.-\mathrm{OCH}_{2}\right), 1.81(3.2 \mathrm{H}-\mathrm{CH}), 1.56-1.25\left(38.4 \mathrm{H}, \mathrm{CH}_{2}\right), 0.98,0.88\left(19.2 \mathrm{H},-\mathrm{CH}_{3}\right)$.

Anal. Found: C, 78.84; $\mathrm{H} 8.85 ; \mathrm{N}, 0.93 ; \mathrm{Br}, 1.68 ; \mathrm{C} / \mathrm{N}$ atomic ratio $=98.9$. The following molecular structure roughly agrees with the found values. Calcd for the following molecular structure: $\mathrm{C}, 79.19 ; \mathrm{H}, 9.20 ; \mathrm{N}, 0.97 ; \mathrm{Br}, 1.73 ; \mathrm{C} / \mathrm{N}$ atomic ratio $=$ 94.9.

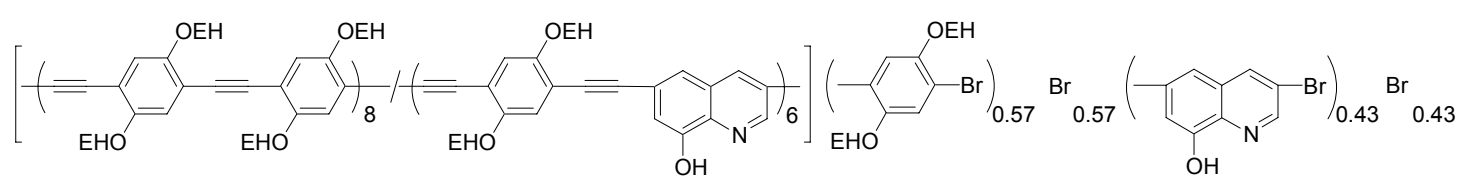

Chart S1 Postulated Molecular Structure of polymer-4b(6/4-OEH)

The PAE-type polymer synthesized by the Pd-catalyzed polycondensation has a 
halogenated terminal group as the main terminal group. ${ }^{11, f}$ The above shown molecular structure gives $M_{\mathrm{n}}$ of 9260 . The $M_{\mathrm{n}}$ corresponds $M_{\mathrm{n}}$ of $9260+$ (molecular weight of the $-\mathrm{SiMe}_{2}{ }^{t} \mathrm{Bu}$ group $) \times 6.43=10000$ of original polymer-1b(6/4-OEH), which roughly agrees with the observed $M_{\mathrm{n}}$ (10400) of polymer-1b(6/4-OEH). The above shown molecular structure somewhat deviates from the feeding ratio between monomer-4 and monomer-b and indicates that monomer-4 had somewhat higher reactivity than monomer-b. The relative reactivity ratio between monomer-4 and monomer-b is calculated as $8 / 6: 6 / 4=1: 1.13$.

Reaction with $\left[\mathbf{A l}(\mathbf{E t})\left(\mathbf{q}^{\prime}\right)_{2}\right]$ After the reaction with $\left[\mathrm{Al}(\mathrm{Et})\left(\mathrm{q}^{\prime}\right)_{2}\right]$, the above shown $\mathrm{C} / \mathrm{N}$ atomic ratio (98.9) of polymer-4b(6/4-OEH) decreased to (59.3), which corresponds to transformation of about $30 \%$ of the 8-quinolinol unit to the Al complex. If two of the 6.438 -quinolinol units in the polymer shown in Chart S1 react with $\left[\mathrm{Al}(\mathrm{Et})\left(\mathrm{q}^{\prime}\right)_{2}\right]$, the degree of the transformation is about $30 \%(=2 / 6.43)$ and the $\mathrm{C} / \mathrm{N}$ atomic ratio becomes 62.4. Determination of the $\mathrm{C} / \mathrm{N}$ ratio and $\mathrm{C} / \mathrm{Al}$ ratio by $\mathrm{ESCA}$ (electron spectroscopy for chemical analysis) was not possible due to low content of $\mathrm{N}$ and $\mathrm{Al}$ in the polymer.

polymer-4a(8/2-OEH), Yield: $88 \%$. 8.81 (0.2H, 2-H-Q), 8.78 (0.2H, 4-H-Q), 7.91 (0.2H, 6-H-Q), 7.53 (0.2H, 3-H-Q), 6.99 (3.6H, Ar-H), $3.90\left(7.2 \mathrm{H},-\mathrm{OCH}_{2}\right), 1.81(3.6 \mathrm{H}$ $-\mathrm{CH}), 1.63-1.25\left(43.2 \mathrm{H}, \mathrm{CH}_{2}\right), 0.98,0.88\left(19.2 \mathrm{H},-\mathrm{CH}_{3}\right)$.

polymer-4b(8/2-OEH), Yield: 92\%. 8.84 (0.2H, 2-H-Q), 8.21 (0.2H, 4-H-Q), 8.05 (0.2H, OH), 7.51 (0.2H, 5-H-Q), 7.27 (0.2H, 7-H-Q), 6.99 (3.6H, Ar-H), 3.90 (7.2H, $\left.-\mathrm{OCH}_{2}\right), 1.81(3.6 \mathrm{H}-\mathrm{CH}), 1.56-1.25\left(43.2 \mathrm{H}, \mathrm{CH}_{2}\right), 0.98,0.88\left(19.2 \mathrm{H},-\mathrm{CH}_{3}\right)$. 


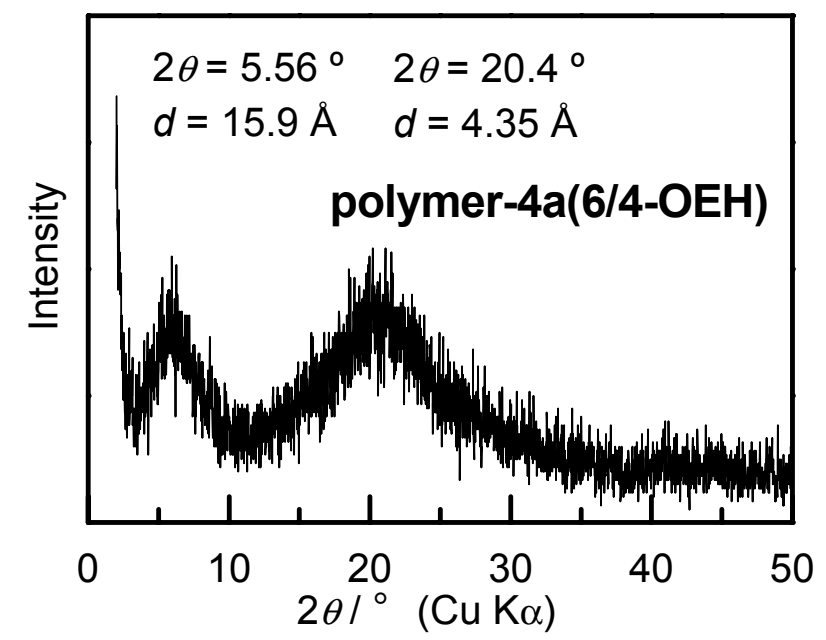

Figure S1 Powder XRD pattern of polymer-4a(6/4-OEH). 


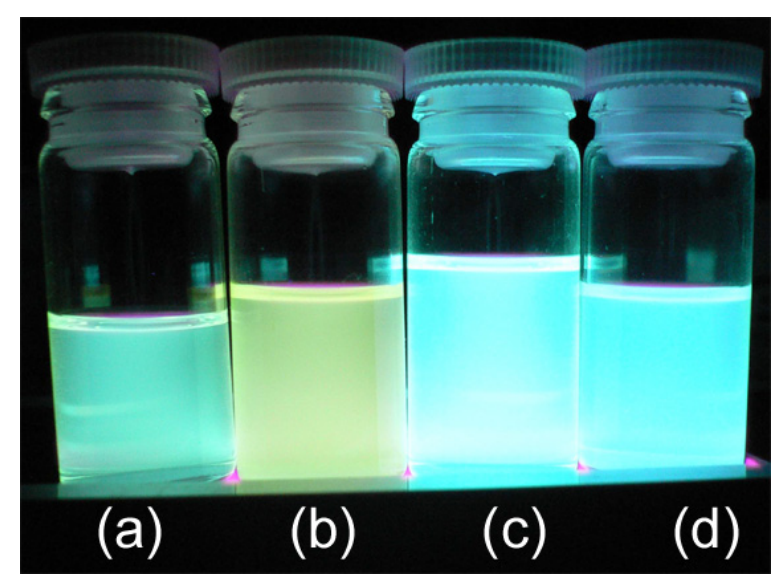

Figure S2. Emission of light from polymer-4a(6/4-OEH) and polymer-4b(6/4-OEH) in the presence of $\mathrm{NaOH}$ in a $4: 1$ (v/v) mixed THF-methanol solution. (a) polymer-4a(6/4-OEH), (b) polymer-4a(6/4-OEH) with $\mathrm{NaOH,}$ (c) polymer-4b(6/4-OEH), and (d) polymer-4b(6/4-OEH) with $\mathrm{NaOH}$. This photograph was taken under irradiation with light at $365 \mathrm{~nm}$. $\mathrm{N}_{2}$ was bubbled in the solution and the sample tube was sealed. 


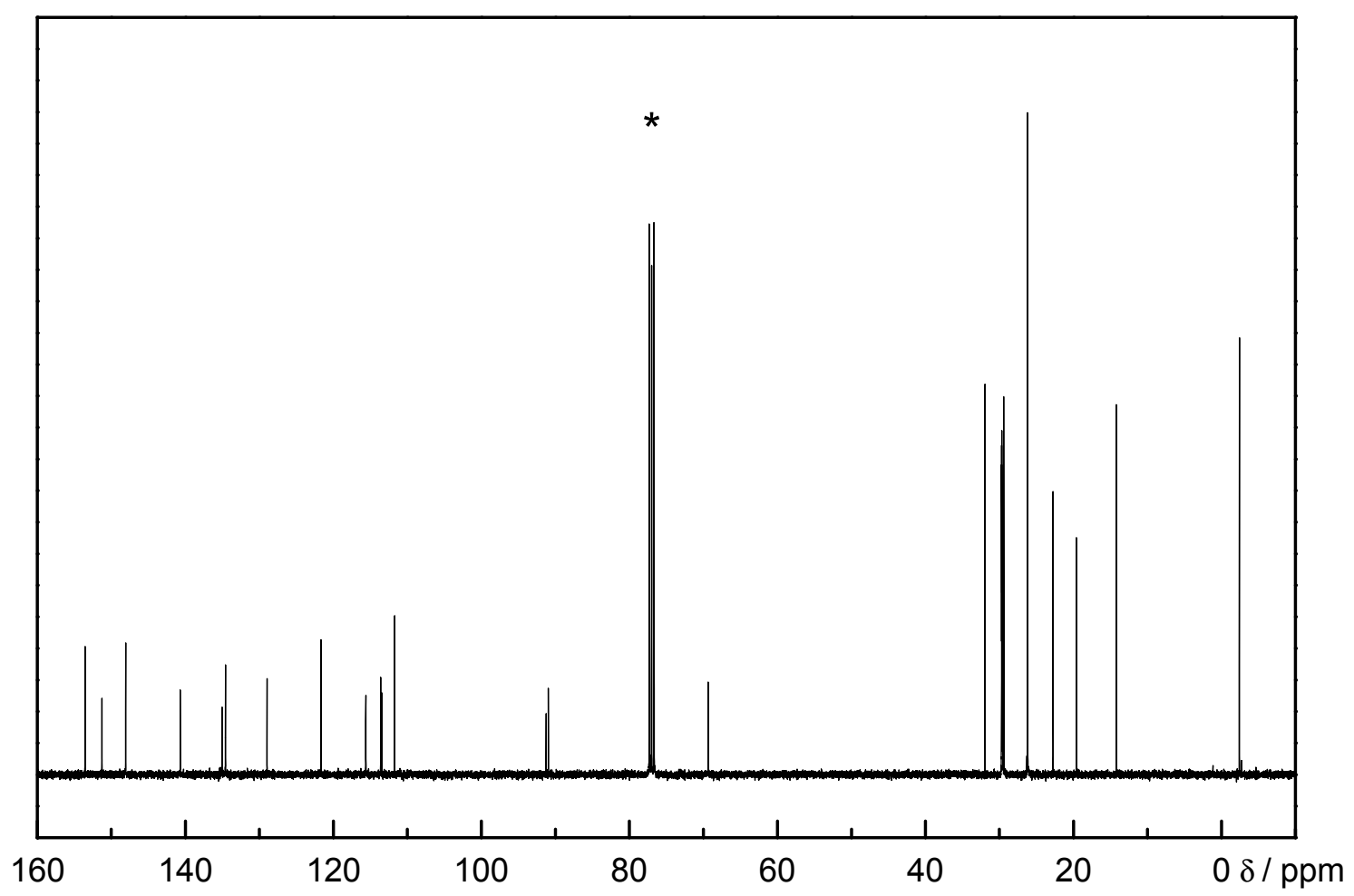

Figure S3. ${ }^{13} \mathrm{C}\left\{{ }^{1} \mathrm{H}\right\}$ NMR spectrum of the model compound $\mathbf{1}$ in $\mathrm{CDCl}_{3}$. The peak with a $*$ is due to solvent impurity $\left(\mathrm{CDCl}_{3}\right)$. 


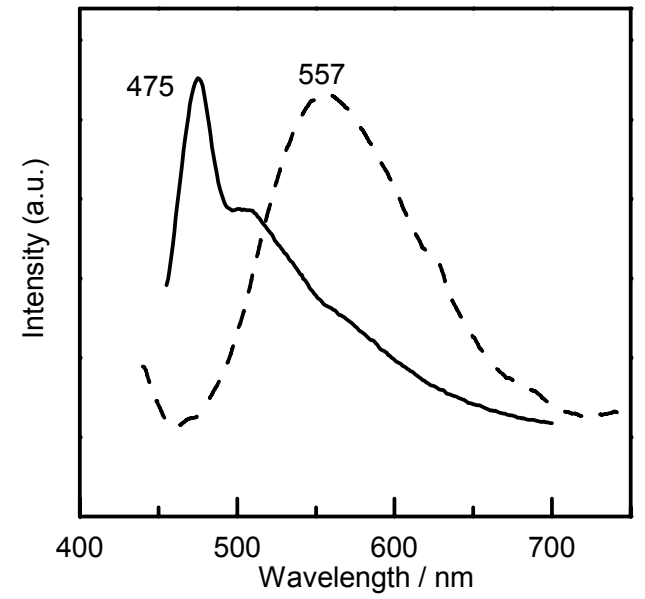

Figure S4. Photoluminescence spectra of a mixture of polymer-4a(6/4-OEH) and $\left[\mathrm{Al}\left(\mathrm{q}^{\prime}\right)_{2}(\mathrm{q})\right] 2$ (in a $1: 1$ molar ratio) in $\mathrm{CHCl}_{3}$ (solid line) and in cast film (dashed line). 